\title{
Association of polycystic ovary syndrome with metabolic syndrome and gestational diabetes: Aggravated complication of pregnancy (Review)
}

\author{
KUI YAO ${ }^{1,2}$, CE BIAN ${ }^{1,2}$ and XIA ZHAO ${ }^{1,2}$ \\ ${ }^{1}$ Key Laboratory of Birth Defects and Related Diseases of Women and Children (Sichuan University), \\ Ministry of Education; ${ }^{2}$ West China Second University Hospital, Chengdu, Sichuan, P.R. China
}

Received March 2, 2017; Accepted June 15, 2017

DOI: $10.3892 /$ etm.2017.4642

\begin{abstract}
Polycystic ovary syndrome (PCOS) affects 5-20\% of the reproductive age women globally. PCOS is diagnosed by the presence of hyperandrogenism, oligo-anovulation, and polycystic morphology of at least one ovary. Insulin resistance (IR), hyperinsulinemia and associated metabolic abnormalities including metabolic syndrome play a significant role in the development of PCOS. The chances of developing MS in PCOS women was shown to increase by almost 14 -fold in patients with increasing body mass index. Even in the absence of overt obesity, a preferential deposition of intra-abdominal fat is noted in PCOS women and this intra-abdominal fat leads to impaired insulin action and functional IR and hyperandrogenism. Functional ovarian hyperandrogenism of ovaries was suggested to be a consequence of IR, which activates androgen synthesizing enzyme, cytochrome p450-c17 $\alpha$-hydroxylase, in ovarian theca cells and causes elevated oxidative stress accompanied by lower antioxidant status in ovaries, which contribute to PCOS pathogenesis. The elevated levels of luteinizing hormone that accompany the early stages of hyperandrogenemia, accelerate ovarian functional deterioration, which is further aggravated by hyperinsulinemia, in PCOS women. The risk of developing gestational diabetes in PCOS women is approximately three times greater, as compared to non-PCOS women, due to IR and hyperinsulinemia. Typical insulin-sensitizing drugs such as metformin, have been used to curtail IR and hyperinsulinemia in pregnant PCOS women, with varying results indicating the complexity of the disease and the need for better controlled studies and additional efforts for PCOS-specific drug discovery.
\end{abstract}

Correspondence to: Dr Xia Zhao, Key Laboratory of Birth Defects and Related Diseases of Women and Children (Sichuan University), Ministry of Education, 20 Section 3, South Renmin Road, Chengdu, Sichuan 610042, P.R. China

E-mail: rxbbjn@163.com

Key words: polycystic ovary syndrome; hyperinsulinemia; cytochrome p 450 -c17 $\alpha$-hydroxylase; functional ovarian hyperandrogenism; metabolic syndrome; gestational diabetes

\section{Contents}

1. Introduction

2. PCOS and obesity

3. Hyperandrogenism and IR in PCOS

4. PCOS and gestational diabetes

5. PCOS and MetS

6. Conclusion

\section{Introduction}

Polycystic ovary syndrome (PCOS) is a very common reproductive endocrinological disorder seen in women, affecting $5-20 \%$ of the reproductive age women globally (1). Insulin resistance (IR) and associated metabolic abnormalities appear to play a significant role in the development of PCOS and in sustaining this disorder $(2,3)$. A vast majority of the affected women also show hyperinsulinemia, developed as a compensatory physiological body need, which in itself contributes to several problems including overweight. Hyperinsulinemia in these patients contributes to the development of metabolic syndrome (MetS), which is a composite of type 2 diabetes, atherosclerosis, obesity and cardiovascular disorders $(4,5)$. The precise etiology of PCOS remains unclear. However, it is suggested that the primary defect lies at the ovarian level or may be a manifestation of hyperinsulinemia that drives elevated androgen production (6). Hyperandrogenism in association with ovulatory dysfunction and polycystic ovarian morphology (PCOM) are common features of PCOS, with the ovaries producing large quantities of androgens (1). This is also accompanied by menstrual disorders (oligo-amenorrhea) (5). The following criteria have been established by several health agencies across the world (National Institutes of Health, European Society of Human Reproduction and Embryology, and American Society of Reproduction Medicine) for the proper diagnosis of PCOS, after eliminating the possibility of other diseases. On the basis of these recommendations, at least two of the following three diagnostic criteria are required for diagnosing PCOS: hyperandrogenism, oligo-anovulation, and polycystic morphology of at least one ovary, as ascertained by ultrasound (minimum 12 follicles of $2-9 \mathrm{~mm}$ in diameter 
or $\geq 10 \mathrm{~cm}^{3}$ ovarian volume). Depending on the presence or absence of ovulation disorders, the phenotypes of PCOS have been separated as the classic PCOS (hyperandrogenism and chronic anovulation, and presence or absence of PCOS) and PCOS with ovulation disorders and polycystic morphology, with IR being evident in both phenotypes $(1,5,7)$.

Apparently, the incidence of MS among PCOS patients seems to be affected also by the geographical region as well as the habits of the patients as it has been recently shown that in Iran the incidence of MS in the Iranian PCOS patients (19.7\%) is less than that seen in United States (33-46\%) (8), India (9) and Brazil (10) and its incidence increases with age and body mass index (BMI), with the most prevalent condition being low/high density lipoprotein-cholesterol (11). On the other hand, the incidence of MS was reported to be lower among European women with PCOS $(12,13)$. It has been suggested that these differences may be due to differences in the criteria used to diagnose MS in these studies. In this review, we have summarized the current knowledge regarding the association of MetS and PCOS and the resulting complications in pregnancy.

\section{PCOS and obesity}

It is well-known that there is elevated risk for type 2 diabetes mellitus, gestational diabetes and other pregnancy-related complications including venous thromboembolism, cerebrovascular and cardiovascular events and endometrial cancer in patients with PCOS (1). The chances of developing MS in PCOS women was shown (8) to increase by almost 14 -fold in patients with BMI in the highest quartile $(\geq 30)$ as compared to those with BMI in the lowest quartile $(<25)$. Fasting insulin level was found to be elevated even in PCOS women without evident MS and it was suggested that the elevated insulin contributes to the elevated androgen production by the ovaries and other complications. Several studies indicated that as much as $60-95 \%$ of PCOS women show IR, which becomes aggravated if accompanied by increased abdominal fat $(14,15)$. However, IR in PCOS women cannot be completely explained by abdominal adiposity and several other factors such as defective glucose, lipid and steroid metabolism, dysregulated insulin signaling and altered adipokine secretion also likely contribute to IR (16). IR and elevated circulating insulin were found to stimulate the theca cells of ovaries to produce and secrete androgens and also to enhance the responsiveness of ovaries to luteinizing hormone $(\mathrm{LH})$ to produce androgens $(5,17)$. In fact, it has been noted that even in the absence of overt obesity, there can be preferential deposition of fat intra-abdominally in PCOS women with normal body weight. This intra-abdominal fat leads to elevated number of small subcutaneous abdominal adipocytes, which contribute to impaired insulin action and thus functional IR and hyperandrogenism (18). Decreased ability of intra-abdominal subcutaneous adipocytes to store and sequester fat in normal weight PCOS women leads to ectopic fat deposition in other tissues such as muscle and liver, and this exerts lipotoxicity and associated IR, contributing to hyperandrogenism $(19,20)$. On the other hand, in overweight PCOS women, adipocytes in the subcutaneous abdominal adipose are large and are not responsive to insulin regulated glucose utilization and also to catecholamine controlled lipolysis and these changes are thought to be androgenmediated (21).

\section{Hyperandrogenism and IR in PCOS}

The association between hyperandrogenism and PCOS stemmed from the observations that elevated levels of free testosterone in plasma of hirsute amenorrheic women actually originate from ovaries (22) and that administration of testosterone resulted in polycystic ovaries in female-to-male transsexuals (23). Those findings led to the hypothesis that hyperandrogenism leads to PCOS. In addition, evidence was presented in some studies that IR is related to hyperandrogenism (24) and that insulin addition to ovaries, in vitro, stimulates them to produce androgens (25) as well as LH (26). These results led to the proposal that hyperinsulinemia as seen in IR conditions contributes to excess androgen production by ovaries. Many of the hyperandrogenic women with classic PCOS display ovarian steroid hyper-responsiveness without any steroidogenic block and also dysregulation of cytochrome $\mathrm{p} 450$-c17 $\alpha$-hydroxylase (27). This type of ovarian dysfunction is known as 'functional ovarian hyperandrogenism', as the steroidogenic response is gonadotropin-dependent (28). Notably, even though the peripheral tissues such as muscle and liver are insulin resistant in PCOS women, the ovaries are very much responsive to both hyperinsulinemia and LH to produce androgens.

Functional ovarian hyperandrogenism was suggested to be a consequence of IR, which causes elevated oxidative stress accompanied by lower antioxidant status in ovaries (29). Increased oxidative stress was found to correlate directly with IR as well as serum testosterone and androstenedione levels (30). IR appears to activate the critical enzyme responsible for the synthesis of androgens, cytochrome p450-c17 $\alpha$-hydroxylase, in ovarian theca cells (Fig. 1), resulting in hyperandrogenism, despite elevated or normal LH secretion (31). Theca cells isolated from polycystic ovaries of classic PCOS patients display elevated expression of several steroidogenic enzymes, specifically, cytochrome P450c17 following long-term cell culture, and also show, that it is characteristic of functional ovarian hyperandrogenism (32). Formation of androgens is controlled by the cytochrome P450c17 enzyme in gonads and also adrenal cortex and its expression is dependent on LH stimulation in ovaries and ACTH in adrenal cortex. Cytochrome P450c17 possesses two activities essential for the generation of androgens: 17-hydroxylase, which converts pregnenolone to 17-hydroxypregnenolone, which is then converted by the 17,20 lyase activity to dehydroepiandrosterone. Dehydroepiandrosterone in turn gives rise to androstenodione and sex steroids. In the theca cells of ovary, cytochrome $\mathrm{P} 450 \mathrm{c} 17$ can also convert progesterone to androstenodione and sex steroids (32). A recent study indicated that in PCOS women with functional ovarian hyperandrogenism, elevated LH:FSH ratio, enhanced oxidative stress and increased levels of free (not total) testosterone correlate with each other positively and that the elevated LH:FSH ratio is a better predictive biomarker for the onset of PCOS in women with functional ovarian hyperandrogenism (30). Elevated levels of androgens cause an inhibition of folliculogenesis that in turn leads to polyfollicular morphology, disturbing menstrual cycle and anovulatory infertility (33). Apparently, androgens have complex effects 


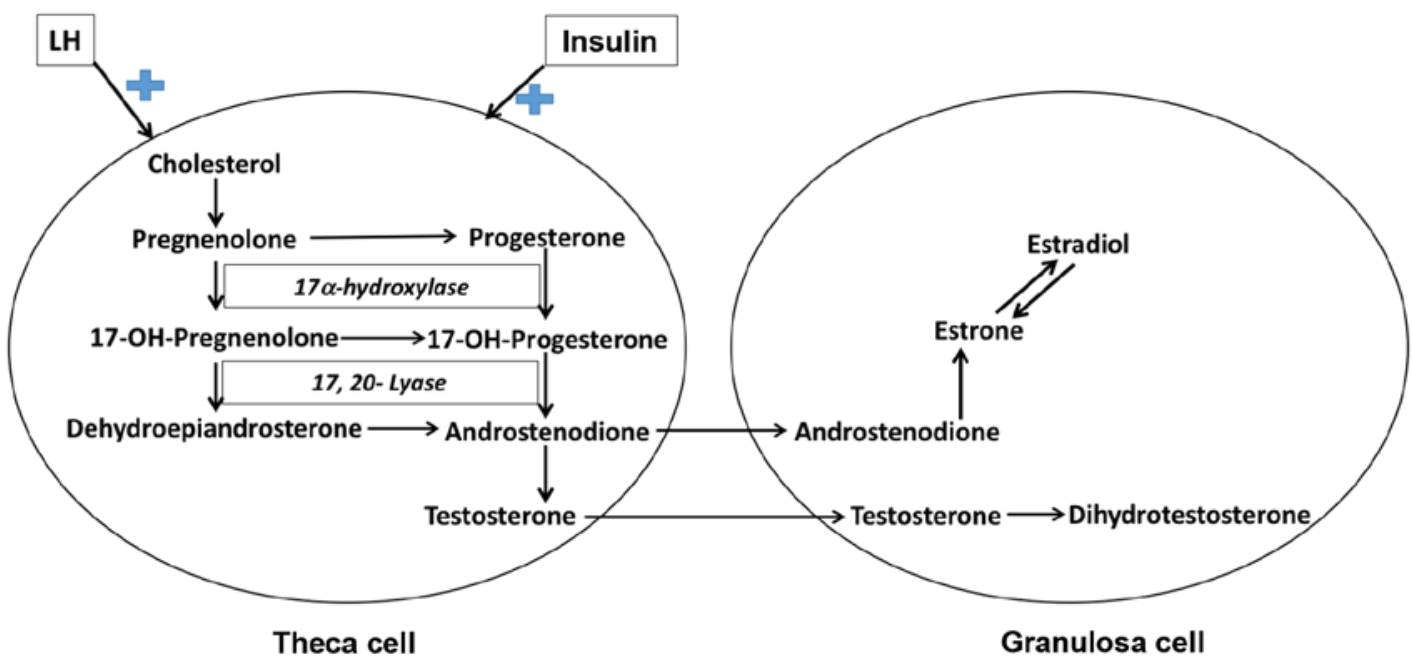

Figure 1. Steroid hormone biosynthetic pathways in ovary. In the ovarian theca cells androgen formation is stimulated by LH via the steroidogenic pathway. Androgen biosynthesis following stimulation by LH is modulated by cytochrome P450c17, which possesses both 17-hydroxylase and 17,20-lyase activities in theca cells. Androstenedione formed in theca cells is also taken up by granulosa cells, where it can contribute to the formation of estrogens. Androstenodione in theca cells is converted to testosterone, which leads to hyperandrogenism. Insulin also stimulates androgen production by theca cells. LH, luteinizing hormone.

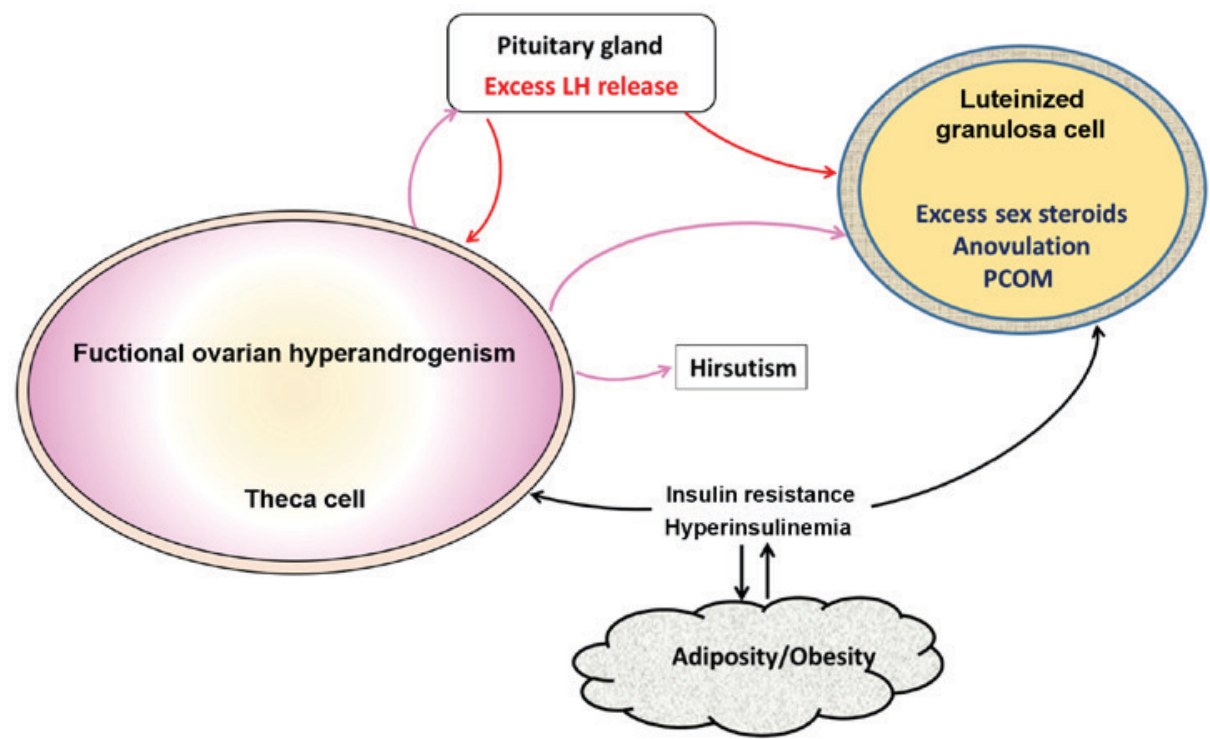

Figure 2. Pathological events in PCOS. Ovarian hyperandrogenism is very common in PCOS and contributes to several abnormalities including hirsutism, oligo-anovulation, and PCOM. LH secretion from pituitary gland is needed for the ovarian androgen production, but other factors such as hyperinsulinism and obesity are also necessary for full-blown pathogenesis of PCOS. Insulin resistance, which is very common in PCOS leads to hyperinsulinemia, which stimulates theca cells and aggravates hyperandrogenism. Excess insulin along with androgen, luteinize granulosa cells prematurely. Adipogenesis is another abnormality resulting from hyperinsulinism. Elevated androgens coming from theca cells in turn stimulate pituitary and cause LH excess, which worsens hyperandrogenism. These changes in granulosa cells further exacerbate PCOM and lead to oligo-anovulation. PCOS, polycystic ovary syndrome; PCOM, polycystic ovarian morphology; LH, luteinizing hormone.

on folliculogenesis at a concentration of $10 \mathrm{ng} / \mathrm{ml}$, testosterone positively affects preantral follicle growth, whereas, when testosterone concentration is elevated to $50 \mathrm{ng} / \mathrm{ml}$, levels seen in the hyperandrogenemia conditions, there is a strong blockade of the follicle growth (34). Testosterone and other androgens can also be synthesized by adrenal cortex, under the stimulation of ACTH and approximately 25\% of the functional ovarian hyperandrogenism cases are actually due to primary functional adrenal hyperandrogenism and contribute to PCOS (35). Thus overall evidence suggests that functional ovarian hyperandrogenism is the underlying causative factor for PCOS and accounts for many of the clinical features of
PCOS such as anovulation, hirsutism and polycystic ovaries. IR and associated hyperinsulinemia aggravate the pathogenic effects of hyperandrogenism. The elevated levels of LH, during the early stages of hyperandrogenemia, accelerate the ovarian functional deterioration, which is further aggravated by accompanying hyperinsulinemia (Fig. 2) (32).

\section{PCOS and gestational diabetes}

Inasmuch as significant populations of PCOS women are insulin resistant and hyperinsulinemic, they are highly prone to develop type 2 diabetes. In fact, a major complication of 
PCOS diagnosis in pregnancy is gestational diabetes (36) and several studies demonstrated increased incidence of gestational diabetes in PCOS women $(37,38)$ and vice versa (39). However, few studies suggested increased BMI to be a better predictor of gestational diabetes than PCOS, raising questions on cause and effect relationship between PCOS and gestational diabetes (40). The risk of developing gestational diabetes in PCOS women is approximately three times greater, as compared to nonPCOS women (41). Treatment of pregnant PCOS women with metformin, an insulin sensitizer drug, commonly used in type 2 diabetic patients, was found to be beneficial as it reduced the high rates of miscarriage usually seen in PCOS women and also the incidence of gestational diabetes $(42,43)$. Although its continuous use is controversial (44), metformin is prescribed to pregnant PCOS women to correct not only the metabolic abnormalities and hyperinsulinemia, but also endocrine disturbances, such as lowering LH and sex-hormone binding globulin levels (45). Metformin is useful as the first-line therapy in PCOS women for inducing ovulation (46). Metformin was suggested to protect against early pregnancy loss in PCOS women, by lowering plasma androgen levels, probably secondary to reduction in insulin levels (47). There is a need to conduct randomized clinical trials with appropriate placebo controls and blinding, with large cohorts, in order to ascertain the beneficial effects of metformin in PCOS women, particularly because this drug does not have any teratogenic effects and has no adverse effects $(45,46)$. It has been suggested that PCOS be considered as a 'prediabetic' condition that is associated with impaired glucose tolerance (with a prevalence of approximately 33\%) and because PCOS women with impaired glucose tolerance develop type 2 diabetes at 5- to 10 -fold higher rate than women without PCOS (48).

\section{PCOS and MetS}

MetS, also known as Syndrome $\mathrm{X}$ is a combination of multiple conditions including central abdominal obesity, hypertension, dyslipidemia and hyperglycemia, all of which are the prime risk factors for cardiovascular diseases. People suffering from MetS display varying degrees of these abnormalities, which primarily result from complex multi-organ interactions of IR, obesity and age (49). Inasmuch as IR is almost globally present in PCOS women, it has been reported that nearly $33 \%$ of adolescents with PCOS develop MetS and this incidence increase to $50 \%$ with age in adults with PCOS $(50,51)$. A recent clinical study with 100 newly diagnosed PCOS women observed that presence of at least two of the following three criteria, viz, hyperandrogenism, oligo/anovulation and polycystic ovaries, poses the highest risk for the development of MetS (52). A recent study has proposed that lipid accumulation product and visceral adiposity index are better markers of IR risk for cardiovascular disease than simple lipid ratios (53). There is a school of thought that suggests that the metabolic abnormalities associated with IR and obesity are probably more important, mechanistically, than hyperandrogenemia for the anovulation in PCOS women $(54,55)$. There is some evidence suggesting women with typical PCOS, i.e., associated hyperandrogenemia, have a heritable component for $\beta$-cell defect and impaired glucose tolerance (56). In PCOS women the extent of IR is often much higher than what is anticipated on the basis of existing adiposity in them. The tissue selective IR together with hyperinsulinism is a major extraovarian contributory factor for the pathogenic alterations seen in PCOS ovaries. Insulin function is preserved in the ovaries of PCOS women despite a state of IR in the body and insulin signaling via insulin receptor in the theca cells appears to mediate the steroidogenic and androgenic effects of insulin (57).

Besides the known association of type 2 diabetes with PCOS as mentioned above, type 1 diabetes also appears to be associated with PCOS and this is particularly because of the very high doses of insulin administered to these patients systemically, for controlling hyperglycemia (58). Almost all the approaches that are commonly employed to correct insulin homeostasis in MS and obese patients, such as lifestyle modification for weight reduction, bariatric surgery, thiazolidinediones and metformin have beneficial effects on ovulation and also control hyperandrogenemia in PCOS women (32). Besides these drugs, of which thiazolidinediones are seldom used, there are inconsistent reports showing efficacy of myo-inositol in improving ovulation and other ovarian functions in PCOS women (59). An important characteristic of MS, the central abdominal obesity, and also occasionally pseudo-Cushing syndrome are noted in PCOS women and appear to be due to hyperinsulinism and possibly also through stimulation of glucocorticoid action and associated $\beta$-cell dysfunction (60). Thus, the overall interrelationship between obesity, IR and hyperandrogenemia together with LH drive the pathogenesis of PCOS.

\section{Conclusion}

PCOS is a very common endocrine disorder affecting a significant proportion of women worldwide and yet there is no effective treatment for this disease. PCOS poses significant risk to pregnant women for loss of pregnancy and/or other associated disorders such as pre-eclampsia and gestational diabetes. PCOS encompasses disturbances in several hormones, including insulin, androgens, and LH. Functional ovarian hyperandrogenism of ovaries, is a consequence of IR, and develops by the activation of androgen synthesis and contributes to PCOS pathogenesis. Despite several common conditions such as diabetes, IR and MetS, seen in non-PCOS obese women, the treatment options available for non-PCOS women do not always work effectively in PCOS women, because of the complexity of the disease. Typical insulin sensitizing drugs such as metformin, have been tried to curtail IR and hyperinsulinemia in pregnant PCOS women, with varying results indicating the complexity of the disease and the need for better controlled studies and additional efforts for PCOS specific drug discovery.

\section{Acknowledgements}

This study was suppoted by Scientific Research Project of Sichuan Medical Association (S16053).

\section{References}

1. Azziz R, Carmina E, Chen Z, Dunaif A, Laven JS, Legro RS, Lizneva D, Natterson-Horowtiz B, Teede HJ and Yildiz BO: Polycystic ovary syndrome. Nat Rev Dis Primers 2: 16057, 2016. 
2. Amato MC, Vesco R, Vigneri E, Ciresi A and Giordano C: Hyperinsulinism and polycystic ovary syndrome (PCOS): role of insulin clearance. J Endocrinol Invest 38: 1319-1326, 2015.

3. Diamanti-Kandarakis E and Dunaif A: Insulin resistance and the polycystic ovary syndrome revisited: an update on mechanisms and implications. Endocr Rev 33: 981-1030, 2012.

4. Ford ES: The metabolic syndrome and mortality from cardiovascular disease and all-causes: findings from the National Health and Nutrition Examination Survey II Mortality Study. Atherosclerosis 173: 309-314, 2004.

5. Polak K, Czyzyk A, Simoncini T and Meczekalski B: New markers of insulin resistance in polycystic ovary syndrome. J Endocrinol Invest 40: 1-8, 2017.

6. Kauffman RP, Baker VM, DiMarino P and Castracane VD Hyperinsulinemia and circulating dehydroepiandrosterone sulfate in white and Mexican American women with polycystic ovary syndrome. Fertil Steril 85: 1010-1016, 2006.

7. Fauser BC, Tarlatzis BC, Rebar RW, Legro RS, Balen AH, Lobo R, Carmina E, Chang J, Yildiz BO, Laven JS, et al: Consensus on women's health aspects of polycystic ovary syndrome (PCOS): the Amsterdam ESHRE/ASRM-sponsored 3rd PCOS consensus workshop group. Fertil Steril 97: 28-38.e25, 2012.

8. Ehrmann DA, Liljenquist DR, Kasza K, Azziz R, Legro RS and Ghazzi MN; PCOS/Troglitazone Study Group: Prevalence and predictors of the metabolic syndrome in women with polycystic ovary syndrome. J Clin Endocrinol Metab 91: 48-53, 2006.

9. Bhattacharya SM: Prevalence of metabolic syndrome in women with polycystic ovary syndrome, using two proposed definitions. Gynecol Endocrinol 26: 516-520, 2010.

10. Soares EM, Azevedo GD, Gadelha RG, Lemos TM and Maranhão TM: Prevalence of the metabolic syndrome and its components in Brazilian women with polycystic ovary syndrome. Fertil Steril 89: 649-655, 2008.

11. Madani T, Hosseini R, Ramezanali F, Khalili G, Jahangiri N, Ahmadi J, Rastegar F and Zolfaghari Z: Metabolic syndrome in infertile women with polycystic ovarian syndrome. Arch Endocrinol Metab 60: 199-204, 2016.

12. Vural B, Caliskan E, Turkoz E, Kilic T and Demirci A: Evaluation of metabolic syndrome frequency and premature carotid atherosclerosis in young women with polycystic ovary syndrome. Hum Reprod 20: 2409-2413, 2005.

13. Carmina E, Napoli N, Longo RA, Rini GB and Lobo RA Metabolic syndrome in polycystic ovary syndrome (PCOS): lower prevalence in southern Italy than in the USA and the influence of criteria for the diagnosis of PCOS. Eur J Endocrinol 154: 141-145, 2006

14. Stepto NK, Cassar S, Joham AE, Hutchison SK, Harrison CL, Goldstein RF and Teede HJ: Women with polycystic ovary syndrome have intrinsic insulin resistance on euglycaemic-hyperinsulaemic clamp. Hum Reprod 28: 777-784, 2013.

15. Moghetti P, Tosi F, Bonin C, Di Sarra D, Fiers T, Kaufman JM Giagulli VA, Signori C, Zambotti F, Dall'Alda M, et al: Divergences in insulin resistance between the different phenotypes of the polycystic ovary syndrome. J Clin Endocrinol Metab 98: E628-E637, 2013.

16. Dumesic DA, Oberfield SE, Stener-Victorin E, Marshall JC, Laven JS and Legro RS: Scientific statement on the diagnostic criteria, epidemiology, pathophysiology, and molecular genetics of polycystic ovary syndrome. Endocr Rev 36: 487-525, 2015

17. Baptiste CG, Battista MC, Trottier A and Baillargeon JP Insulin and hyperandrogenism in women with polycystic ovary syndrome. J Steroid Biochem Mol Biol 122: 42-52, 2010.

18. Dumesic DA, Akopians AL, Madrigal VK, Ramirez E, Margolis DJ, Sarma MK, Thomas AM, Grogan TR, Haykal R, Schooler TA, et al: Hyperandrogenism accompanies increased intra-abdominal fat storage in normal weight polycystic ovary syndrome women. J Clin Endocrinol Metab 101: 4178-4188, 2016.

19. de Zegher F, Lopez-Bermejo A and Ibáñez L: Adipose tissue expandability and the early origins of PCOS. Trends Endocrinol Metab 20: 418-423, 2009.

20. Unger RH, Clark GO, Scherer PE and Orci L: Lipid homeostasis, lipotoxicity and the metabolic syndrome. Biochim Biophys Acta 1801: 209-214, 2010

21. Mannerås-Holm L, Leonhardt H,Kullberg J, Jennische E, Odén A, Holm G, Hellström M, Lönn L, Olivecrona G, Stener-Victorin E, et al: Adipose tissue has aberrant morphology and function in PCOS: enlarged adipocytes and low serum adiponectin, but not circulating sex steroids, are strongly associated with insulin resistance. J Clin Endocrinol Metab 96: E304-E311, 2011.
22. Rosenfield RL, Ehrlich EN and Cleary RE: Adrenal and ovarian contributions to the elevated free plasma androgen levels in hirsute women. J Clin Endocrinol Metab 34: 92-98, 1972.

23. Futterweit W and Deligdisch L: Histopathological effects of exogenously administered testosterone in 19 female to male transsexuals. J Clin Endocrinol Metab 62: 16-21, 1986.

24. Dunaif A, Segal KR, Futterweit W and Dobrjansky A: Profound peripheral insulin resistance, independent of obesity, in polycystic ovary syndrome. Diabetes 38: 1165-1174, 1989.

25. Barbieri RL, Makris A, Randall RW, Daniels G, Kistner RW and Ryan KJ: Insulin stimulates androgen accumulation in incubations of ovarian stroma obtained from women with hyperandrogenism. J Clin Endocrinol Metab 62: 904-910, 1986.

26. Cara JF and Rosenfield RL: Insulin-like growth factor I and insulin potentiate luteinizing hormone-induced androgen synthesis by rat ovarian thecal-interstitial cells. Endocrinology 123 : 733-739, 1988.

27. Rosenfield RL, Barnes RB, Cara JF and Lucky AW: Dysregulation of cytochrome P450c 17 alpha as the cause of polycystic ovarian syndrome. Fertil Steril 53: 785-791, 1990.

28. Ehrmann DA, Rosenfield RL, Barnes RB, Brigell DF and Sheikh Z: Detection of functional ovarian hyperandrogenism in women with androgen excess. N Engl J Med 327: 157-162, 1992.

29. González F, Rote NS, Minium J and Kirwan JP: Reactive oxygen species-induced oxidative stress in the development of insulin resistance and hyperandrogenism in polycystic ovary syndrome. J Clin Endocrinol Metab 91: 336-340, 2006.

30. Suresh S and Vijayakumar T: Correlations of insulin resistance and serum testosterone levels with LH:FSH ratio and oxidative stress in women with functional ovarian hyperandrogenism. Indian J Clin Biochem 30: 345-350, 2015.

31. Nisenblat V and Norman RJ: Androgens and polycystic ovary syndrome. Curr Opin Endocrinol Diabetes Obes 16: 224-231, 2009.

32. Rosenfield RL and Ehrmann DA: The pathogenesis of polycystic ovary syndrome (PCOS): the hypothesis of pcos as functional ovarian hyperandrogenism revisited. Endocr Rev 37: 467-520, 2016.

33. Azziz R: Diagnostic criteria for polycystic ovary syndrome: a reappraisal. Fertil Steril 83: 1343-1346, 2005.

34. Rodrigues JK, Navarro PA, Zelinski MB, Stouffer RL and Xu J: Direct actions of androgens on the survival, growth and secretion of steroids and anti-Müllerian hormone by individual macaque follicles during three-dimensional culture. Hum Reprod 30: 664-674, 2015

35. Azziz R, Carmina E, Dewailly D, Diamanti-Kandarakis E, Escobar-Morreale HF, Futterweit W, Janssen OE, Legro RS, Norman RJ, Taylor AE, et al; Task Force on the Phenotype of the Polycystic Ovary Syndrome of The Androgen Excess and PCOS Society: The androgen excess and PCOS society criteria for the polycystic ovary syndrome: the complete task force report. Fertil Steril 91: 456-488, 2009.

36. Homburg R: Pregnancy complications in PCOS. Best Pract Res Clin Endocrinol Metab 20: 281-292, 2006.

37. Bjercke S, Dale PO, Tanbo T, Storeng R, Ertzeid G and Abyholm T: Impact of insulin resistance on pregnancy complications and outcome in women with polycystic ovary syndrome. Gynecol Obstet Invest 54: 94-98, 2002.

38. Weerakiet S, Srisombut C, Rojanasakul A, Panburana P, Thakkinstian A and Herabutya Y: Prevalence of gestational diabetes mellitus and pregnancy outcomes in Asian women with polycystic ovary syndrome. Gynecol Endocrinol 19: 134-140, 2004.

39. Anttila L, Karjala K, Penttilä RA, Ruutiainen K and Ekblad U: Polycystic ovaries in women with gestational diabetes. Obstet Gynecol 92: 13-16, 1998.

40. Turhan NO, Seçkin NC, Aybar F and Inegöl I: Assessment of glucose tolerance and pregnancy outcome of polycystic ovary patients. Int J Gynaecol Obstet 81: 163-168, 2003.

41. Qin JZ, Pang LH, Li MJ, Fan XJ, Huang RD and Chen HY: Obstetric complications in women with polycystic ovary syndrome: a systematic review and meta-analysis. Reprod Biol Endocrinol 11: 56, 2013.

42. Glueck CJ, Wang P, Goldenberg N and Sieve-Smith L: Pregnancy outcomes among women with polycystic ovary syndrome treated with metformin. Hum Reprod 17: 2858-2864, 2002.

43. Glueck CJ, Wang P, Kobayashi S, Phillips H and Sieve-Smith L: Metformin therapy throughout pregnancy reduces the development of gestational diabetes in women with polycystic ovary syndrome. Fertil Steril 77: 520-525, 2002. 
44. Ghazeeri GS, Nassar AH, Younes Z and Awwad JT: Pregnancy outcomes and the effect of metformin treatment in women with polycystic ovary syndrome: an overview. Acta Obstet Gynecol Scand 91: 658-678, 2012.

45. Nawaz FH, Khalid R, Naru T and Rizvi J: Does continuous use of metformin throughout pregnancy improve pregnancy outcomes in women with polycystic ovarian syndrome? J Obstet Gynaecol Res 34: 832-837, 2008

46. Costello MF and Eden JA: A systematic review of the reproductive system effects of metformin in patients with polycystic ovary syndrome. Fertil Steril 79: 1-13, 2003.

47. Jakubowicz DJ, Iuorno MJ, Jakubowicz S, Roberts KA and Nestler JE: Effects of metformin on early pregnancy loss in the polycystic ovary syndrome. J Clin Endocrinol Metab 87: 524-529, 2002

48. Ben-Haroush A, Yogev Y and Hod M: Epidemiology of gestational diabetes mellitus and its association with type 2 diabetes. Diabet Med 21: 103-113, 2004

49. Eckel RH, Grundy SM and Zimmet PZ: The metabolic syndrome. Lancet 365: 1415-1428, 2005.

50. Rossi B, Sukalich S, Droz J, Griffin A, Cook S, Blumkin A, Guzick DS and Hoeger KM: Prevalence of metabolic syndrome and related characteristics in obese adolescents with and without polycystic ovary syndrome. J Clin Endocrinol Metab 93 : 4780-4786, 2008.

51. Apridonidze T, Essah PA, Iuorno MJ and Nestler JE: Prevalence and characteristics of the metabolic syndrome in women with polycystic ovary syndrome. J Clin Endocrinol Metab 90 : 1929-1935, 2005

52. Bil E, Dilbaz B, Cirik DA, Ozelci R, Ozkaya E and Dilbaz S: Metabolic syndrome and metabolic risk profile according to polycystic ovary syndrome phenotype. J Obstet Gynaecol Res 42: 837-843, 2016

53. Abruzzese GA, Cerrone GE, Gamez JM, Graffigna MN, Belli S, Lioy G, Mormandi E, Otero P, Levalle OA and Motta AB: Lipid accumulation product (LAP) and visceral adiposity index (VAI) as markers of insulin resistance and metabolic associated disturbances in young argentine women with polycystic ovary syndrome. Horm Metab Res 49: 23-29, 2016.
54. Turkmen S, Ahangari A and Bäckstrom T: Roux-en-y gastric bypass surgery in patients with polycystic ovary syndrome and metabolic syndrome. Obes Surg 26: 111-118, 2016.

55. Legro RS, Dodson WC, Kris-Etherton PM, Kunselman AR, Stetter CM, Williams NI, Gnatuk CL, Estes SJ, Fleming J, Allison KC, et al: Randomized controlled trial of preconception interventions in infertile women with polycystic ovary syndrome. J Clin Endocrinol Metab 100: 4048-4058, 2015.

56. Colilla S, Cox NJ and Ehrmann DA: Heritability of insulin secretion and insulin action in women with polycystic ovary syndrome and their first degree relatives. J Clin Endocrinol Metab 86: 2027-2031, 2001.

57. Wu S, Divall S, Nwaopara A, Radovick S, Wondisford F, Ko C and Wolfe A: Obesity-induced infertility and hyperandrogenism are corrected by deletion of the insulin receptor in the ovarian theca cell. Diabetes 63: 1270-1282, 2014.

58. Codner E and Escobar-Morreale HF: Clinical review: hyperandrogenism and polycystic ovary syndrome in women with type 1 diabetes mellitus. J Clin Endocrinol Metab 92: 1209-1216, 2007.

59. Facchinetti F, Bizzarri M, Benvenga S, D'Anna R, Lanzone A, Soulage C, Di Renzo GC, Hod M, Cavalli P, Chiu TT, et al: Results from the International Consensus Conference on myo-inositol and d-chiro-inositol in obstetrics and gynecology: the link between metabolic syndrome and PCOS. Eur J Obstet Gynecol Reprod Biol 195: 72-76, 2015.

60. Ehrmann DA, Breda E, Corcoran MC, Cavaghan MK, Imperial J, Toffolo G, Cobelli C and Polonsky KS: Impaired beta-cell compensation to dexamethasone-induced hyperglycemia in women with polycystic ovary syndrome. Am J Physiol Endocrinol Metab 287: E241-E246, 2004. 